\title{
Probing regolith-covered surfaces in low gravity
}

\author{
Jonathan E. Kollmer ${ }^{1,2, *}$, Jack Featherstone ${ }^{1}$, Robert Bullard ${ }^{1}$, Tristan Emm ${ }^{1}$, Anna Jackson ${ }^{1}$, Riley Reid ${ }^{1}$, Sean \\ Shefferman $^{3}$, Adrienne Dove ${ }^{3}$, Joshua Colwell ${ }^{3}$, and Karen E. Daniels ${ }^{1}$ \\ ${ }^{1}$ Dept. of Physics, North Carolina State University, Raleigh, NC, 27695, USA \\ ${ }^{2}$ Fakultät für Physik, Universität Duisburg-Essen, 47057 Duisburg, Germany \\ ${ }^{3}$ Department of Physics, University of Central Florida, Orlando, FL, 32816, USA
}

\begin{abstract}
The surfaces of many planetary bodies, including asteroids, moons, and planets, are composed of rubble-like grains held together by varying levels of gravitational attraction and cohesive forces. Future instrumentation for operation on, and interacting with, such surfaces will require efficient and effective design principles and methods of testing. Here we present results from the EMPANADA experiment (Ejecta-Minimizing Protocols for Applications Needing Anchoring or Digging on Asteroids) which flew on several reduced gravity parabolic flights. EMPANADA studies the effects of the insertion of a flexible probe into a granular medium as a function of ambient gravity. This is done for an idealized 2D system as well as a more realistic 3D sample. To quantify the dynamics inside the 2D granular material we employ photoelasticity to identify the grain-scale forces throughout the system, while in 3D experiments we use simulated regolith. Experiments were conducted at three different levels of gravity: martian, lunar, and microgravity. In this work, we demonstrate that the photoelastic technique provides results that complement traditional load cell measurements in the 2D sample, and show that the idealized system exhibits similar behaviour to the more realistic 3D sample. We note that the presence of discrete, stick-slip failure events depends on the gravitational acceleration.
\end{abstract}

\section{Introduction}

Understanding how grains rearrange when probed is integral to developing safe and efficient techniques for sample collection and anchoring on planetary bodies. The surfaces of asteroids, moons, and even certain planets are characteristically described as a weakly interacting collection of heterogeneous grains. Such bodies include our moon [1], as well as numerous near-Earth asteroids like Itokawa, Bennu, and Ryugu, which have recently been visited by unmanned spacecraft and confirmed to be composed of loosely bound rubble [2, 3].

There have been a number of recent studies investigating low-velocity impacts on granular surfaces in low gravity $[4,5]$. These studies, however, are mostly focused on fundamental properties of asteroid and planetary surfaces and the nature of granular collisions, rather than the applications of digging or anchoring that are relevant to low-gravity sampling missions. While the dynamics of a driven flexible probe have yet to be extensively explored in a low gravity environment, there has been recent interest in the insertion of flexible probes into granular materials on Earth [6, 7].

In this work, we present early results from the $2 \mathrm{D}$ and 3D EMPANADA (Ejecta-Minimizing Protocols for Applications Needing Anchoring or Digging on Asteroids) experiment. EMPANADA [8] investigates the insertion of a flexible probe [9] into a granular medium, simulating

*e-mail: jonathan.kollmer@uni-due.de

A video is available at https://doi.org/10.48448/946w-ez57 a rubble covered surface, under various levels of gravity. Three different levels of gravity (martian-, lunar-, and microgravity ) were achieved during a parabolic flight campaign conducted by Zero Gravity Corporation.

The experimental setup is shown in Figure 1 and centers around a flexible probe mounted on a slider driven by a stepper motor. To provide a full view of the dynamics, EMPANADA-2D uses $\sim 1 \mathrm{~cm}$ diameter photoelastic discs. These photoelastic particles allow a camera to capture force information on a particle level $[10,11]$ and therefore give insight into the granular dynamics of the system. The photoelastic data is complemented by a load cell mounted to the insertion device. To compare the experiment to a more realistic environment, EMPANADA3D conducts similar experiments using a regolith simulant [12] in a vacuum.

In this paper, we show that globally averaged parameters taken from photoelastic response images, such as the brightness of each frame, exhibit the same response as the load cell. We further show that the qualitative load behavior is similar for the $2 \mathrm{D}$ and $3 \mathrm{D}$ system. This qualitative agreement validates the use of experimentally moreaccessible 2D systems for providing details about insertion dynamics. Lastly, we show that the level of gravity changes the response of the granular bed, the details of which are provided in [8]. 


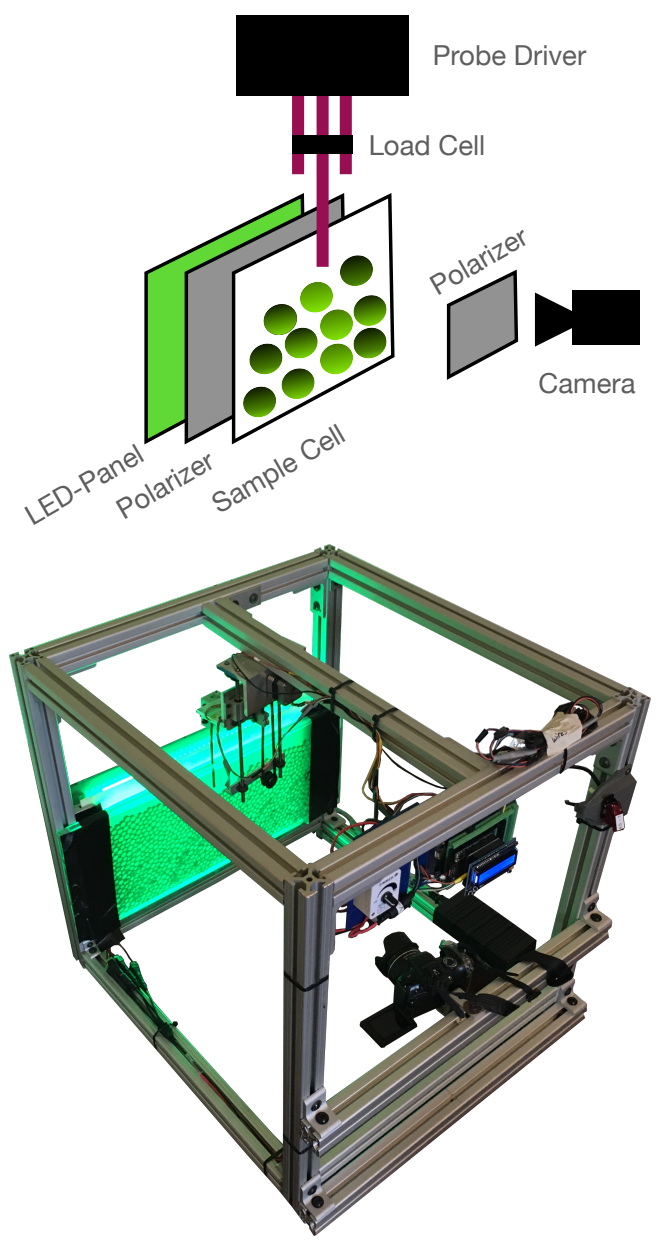

Figure 1: The EMPANADA-2D experimental setup. Top: Schematic drawing. Bottom: Photograph of the assembled apparatus to be mounted into the ZERO-G aircraft. The plane-mountable frame contains the probing mechanism, the 2D-sample of backlit photoelastic particles, and a digital camera. Four EMPANADA-3D units (see Fig. 5) were mounted below.

\section{Results}

To evaluate weather the photoelastic technique can sufficiently complement traditional load measurements, we look at the time derivative of the brightness as a metric for how the medium is changing throughout the insertion process. This is done using a discretized derivative stencil on the average frame brightness.

Due to safety considerations, the particle enclosure was constructed from acrylic sheets. Unfortunately, these act as fractional waveplates, slightly rotating the polarization of the light transmitted through the particles and therefore disturbing the photoelastic response signal. This effect manifests as force chains of particular orientations appearing dark (rather than bright) in the recorded images. We accounted for this issue by pre-processing the images so as to highlight the force chains regardless of their brightness in the raw images.

After processing the images, we compare the results to those made with the load cell readings on the probe. (a)

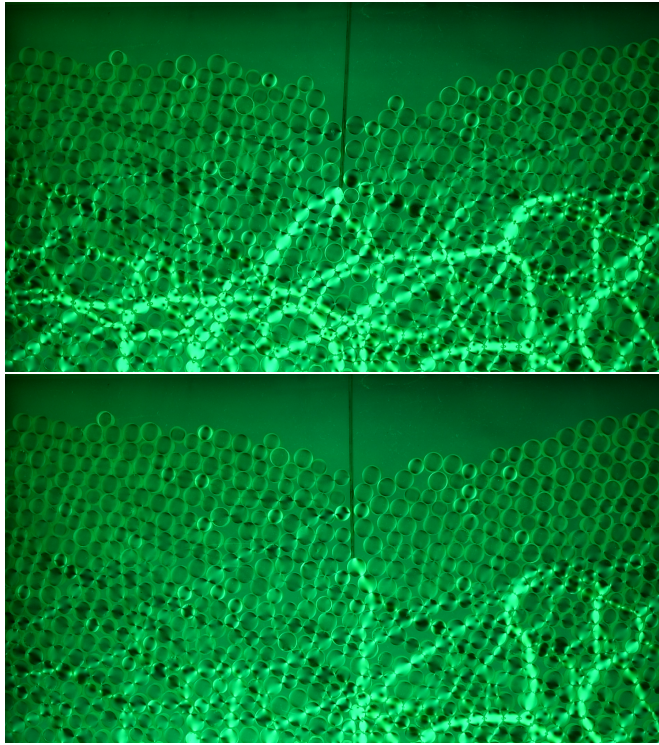

(c)

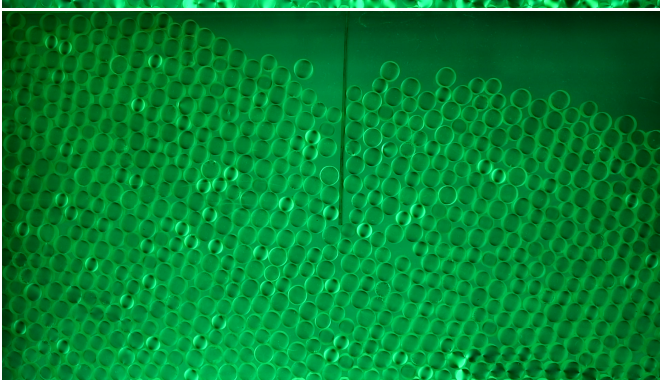

Figure 2: Video frames showing the photoelastic response to probe insertion from the (a) Martian, (b) lunar, and (c) microgravity trials. The Martian and lunar experiments show easily-recognizable force chains.

This comparison, shown in Figs. 3a, 3b, and 3c, demonstrates that the time derivative of the global image brightness best identifies the slip events, while the $G^{2}$ analysis [11] more closely mimics the load cell readings. Therefore, in circumstances where the aim is to count slip events [8], brightness measurements can suffice.

Fig. 4a shows a comparison of the average brightness (accumulated strength of contact forces) during probe insertion for 3 different trials, covering martian-, lunar-, and microgravity. Globally, higher g-levels result in a higher base load; this is visible as an offset in the magnitude of the force data. Additionally, runs at higher gravitational acceleration tend to give a more sharply-peaked average brightness during the insertion process, corresponding to stronger forces throughout the granular bed. Martian and lunar gravity show easily identifiable force events, in the form of local maxima, which we identify as stick-slip behavior. As a general trend, we observe a more pronounced sick slip behaviour for higher gravity levels. While there are features in the microgravity data that could be considered as discrete events, they don't seem to represent the dominant pathway for spatial reconfiguration.

A more quantitative analysis of both $2 \mathrm{D}$ experiments is the subject of current research and will be detailed in a separate publication [8]. 


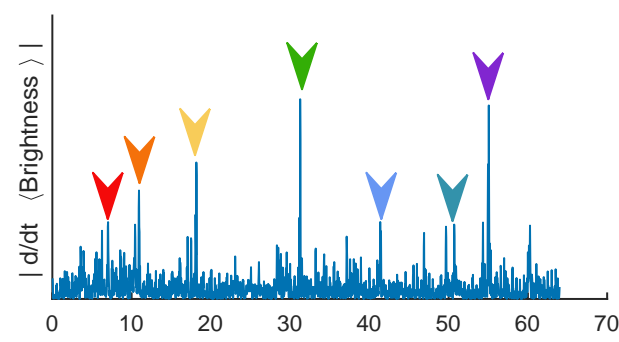

(a)

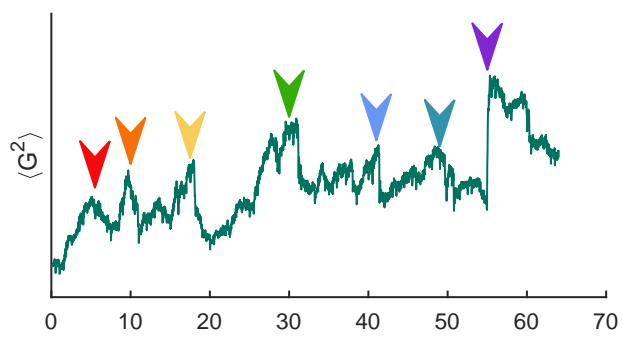

(b)

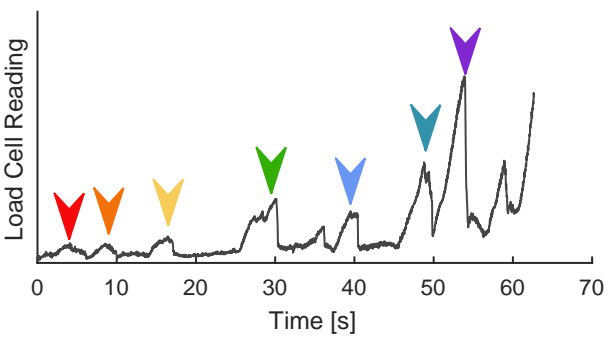

(c)

Figure 3: A comparison between the granular activity as measured by: (a) the time derivative of the average frame brightness, (b) the average $G^{2}$ of each frame, and (c) the load cell readings on the probe. Arrow colors indicate the same physical event as demonstrated by each metric.

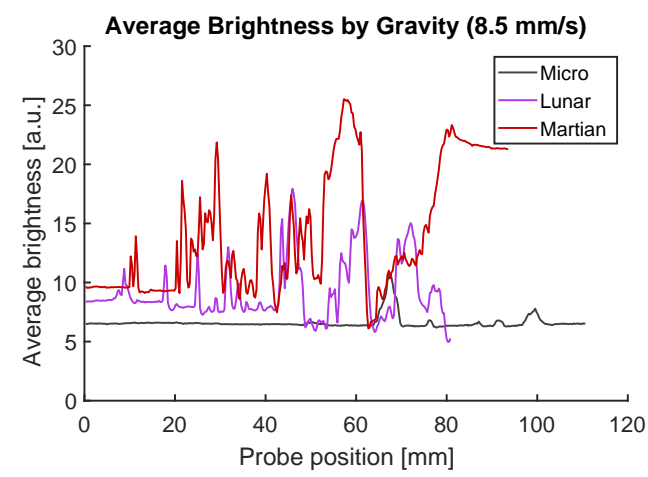

Figure 4: The average brightness of the total photoelastic response in martian, lunar and microgravity. The probe insertion speed was $8.5 \mathrm{~mm} / \mathrm{s}$ in all cases. Note that the offset between experiments stems from the different base loads of the granular system due to the different gravities.

\section{Comparing 2D to 3D data}

One of the aims of this experiment was to determine the extent to which idealized 2D experiments can serve as a proxy for more realistic 3D experiments. Four EMPANADA-3D experiments, with probes of four different stiffnesses, flew on the same parabolic flights as EMPANADA-2D. In these experiments, a stepper motor drives a probe into a $3 \mathrm{D}$ system of finely powdered re-

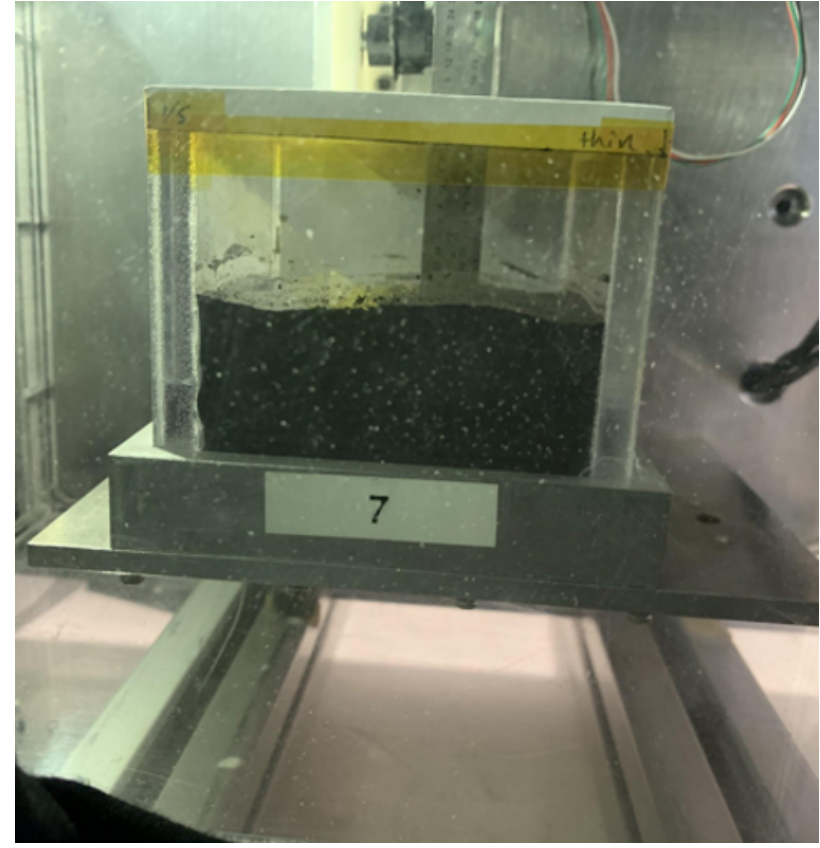

Figure 5: Photograph of the 3D experiment mounted into the Zero-G plane

golith simulant [12]. while a load cell records the force applied onto the probe. This experiment was housed in an evacuated box to better mimic an extraterrestrial environment. A picture of the experiment can be seen in Fig. 5 .

Due to experimental issues, an absolute force calibration of the load cell was not possible. Instead, in order to make a bulk comparison, we rescale all data series (at a variety of grains, speeds and probe stiffnesses) by their mean loads, in order to nondimensionalize the data. In the analysis that follows, the load on the EMPANADA-3D simulated regolith bed is measured directly from the voltage on the load cell attached to the probe. For EMPANADA-2D experiments, the load on the photoelastic granular bed is measured from the local spatial gradient $\left(G^{2}\right.$ method) of the image brightness [11].

Fig. 6 shows the direct comparison, where the "Dataset" axis sequentially displays the results from each flown parabola, grouped by gravity level according to the dashed lines. Data points in the same numbered dataset were collected on the same parabola. With the exception of a few outliers in the microgravity data (assumed to be from g-jitter), the datasets all collectively follow the same trend. This qualitative agreement of $2 \mathrm{D}$ and $3 \mathrm{D}$ data indicates the success of the 2D photoelastic model system as a proxy for capturing the granular behaviors in more realistic systems. However, one needs to apply proper caution: 2D and 3D granular materials are known to have different behaviours (e.g. arching etc.). Further study would elucidate the generality of these results.

\section{Conclusions}

We've shown that techniques borrowed from Earth-based granular physics can be used to study regolith dynamics on 


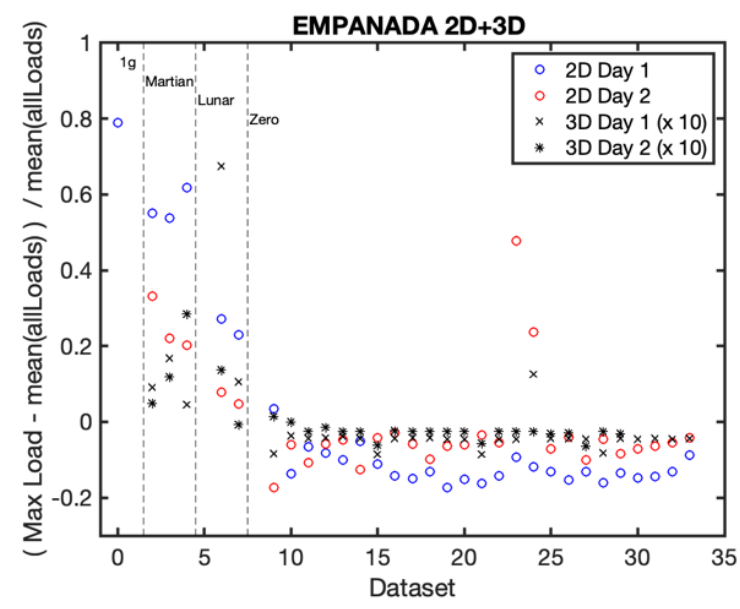

Figure 6: Comparison of load variations during probing of the $2 \mathrm{D}$ and $3 \mathrm{D}$ datasets.

planetary bodies. We successfully constructed an experiment that can test insertion dynamics in a variable gravity environment and have shown that the photoelastic response and load cell readings on the intruder are in good correspondence. We further demonstrated that load cell data from a 3D experiment with realistic materials yield similar trends in load response. These measurements validate our approach to use 2D systems with idealized particles to study the granular physics of material response to external perturbation at low gravities. For example, it could lead to experiments where we properly calibrate these results with $2 \mathrm{D}$ experiments in the lab, obtained from frictionless slightly-inclined planes [9].

We have further observed that, depending on ambient gravity, the discrete nature (stick slip) of the granular bed becomes more pronounced at higher gravity, and vanishes in microgravity. This clear dependence suggests that more research is warranted to investigate the nature of this transition as a function of $g$.

\section{Acknowledgements:}

We acknowledge Jacob Anthony, Emily D'Elia, Gillian Gomer and Cody Schultz for help with EMPANADA3D. This work was funded by NASA Grant number 80NSSC18K0269, with undergraduate student funding from both the NC State Office of Undergraduate Research and the NC State Provost's Professional Experience Program. JK acknowledges funding from the German Aerospace Center DLR under grant number 50WM1943.

\section{References}

[1] D.B. Nash, J.W. Salisbury, J.E. Conel, P.G. Lucey, P.R. Christensen, J. Geophys. Res. Planets 98, 23535 (1993)

[2] D.N. DellaGiustina, J.P. Emery, D.R. Golish, B. Rozitis, C.A. Bennett, K.N. Burke, R.L. Ballouz, K.J. Becker, P.R. Christensen, C.Y. Drouet d'Aubigny et al., Nat. Astron. 3, 341 (2019)

[3] S. Watanabe, M. Hirabayashi, N. Hirata, N. Hirata, R. Noguchi, Y. Shimaki, H. Ikeda, E. Tatsumi, M. Yoshikawa, S. Kikuchi et al., Science 364, 268 (2019)

[4] J. Brisset, J. Colwell, A. Dove, S. Abukhalil, C. Cox, N. Mohammed, Prog. Earth Planet. Sci. 5, 1 (2018)

[5] Brisset, J., Cox, C., Anderson, S., Hatchitt, J., Madison, A., Mendonca, M., Partida, A., Remie, D., A\&A 642, A198 (2020)

[6] N. Algarra, P.G. Karagiannopoulos, A. Lazarus, D. Vandembroucq, E. Kolb, Phys. Rev. E 97, 022901 (2018)

[7] A.R. Mojdehi, B. Tavakol, W. Royston, D.A. Dillard, D.P. Holmes, Extreme Mech. Lett. 9, Part 1, 237 (2016)

[8] J. Featherstone, R. Bullard, T. Emm, A. Jackson, R. Reid, S. Shefferman, A. Dove, J. Colwell, J.E. Kollmer, K.E. Daniels, arXiv:2011.12890 (2020)

[9] J.E. Kollmer, S. Lindauer, K.E. Daniels, ASCE Earth and Space Conference (2016)

[10] K.E. Daniels, J.E. Kollmer, J.G. Puckett, Rev. Sci. Instrum. 88, 051808 (2017)

[11] A. Abed Zadeh, J. Barés, T.A. Brzinski, K.E. Daniels, J. Dijksman, N. Docquier, H.O. Everitt, J.E. Kollmer, O. Lantsoght, D. Wang et al., Granul. Matter 21, 83 (2019)

[12] D.T. Britt, K.M. Cannon, K. Donaldson Hanna, J. Hogancamp, O. Poch, P. Beck, D. Martin, J. Escrig, L. Bonal, P.T. Metzger, Meteorit Planet Sci. 54, 2067 (2019) 